\title{
A experimentação na construção de conceitos em físico-química ${ }^{1}$
}

\section{Experimentation on the construction of concepts in physical chemistry}

\author{
DOI: $10.46814 /$ lajdv3n2-007
}

Recebimento dos originais: 23/12/2020

Aceitação para publicação: 26/02/2021

\section{Fernanda P. Zabiela \\ Mestre}

Pós Graduação em Educação em Ciências, Universidade Federal do Rio Grande do Sul Rua Ramiro Barcelos, 2600, Prédio Anexo, 90035-003, Santa Cecília, Porto Alegre, RS

E-mail: fe.ponticelli@hotmail.com

\section{Andréia M. Zucolotto}

Doutora

Campus Porto Alegre, Instituto Federal do Rio Grande do Sul Rua Coronel Vicente, 281, 90030-041, Centro Histórico, Porto Alegre, RS

E-mail: andreia.zucolotto@poa.ifrs.edu.br

\author{
Alexandre Beluco \\ Doutor \\ Instituto de Pesquisas Hidráulicas, Universidade Federal do Rio Grande do Sul \\ Av Bento Gonçalves, 9500, Caixa Postal 9500, 91501-970, Agronomia, Porto Alegre, RS \\ E-mail: albeluco@iph.ufrgs.br
}

\section{RESUMO}

O presente artigo é fruto de uma estudo realizado com uma turma de segundo módulo da disciplina de Físico Química de um curso técnico em química de uma escola privada da região metropolitana de Porto Alegre, RS. Foi desenvolvida uma atividade experimental com o objetivo de construção de conceitos relativos as grandezas massa, volume e concentração. Foram aplicados testes para avaliação de conhecimentos antes e depois do desenvolvimento dessa atividade experimental. Os resultados anteriores a essa atividade foram relativamente ruins, com nota mínima 1,0, e com média igual a 6,6. Os resultados foram avaliados estatisticamente mediante a aplicação de análise de variância, que revelou uma melhora nos resultados dos testes com significância de 5\%. Os novos resultados apresentaram nota mínima 4,0 e média 8,9.

Palavra chave: massa, volume, concentração, grandezas, dificuldades, aprendizagens, experiências.

\section{ABSTRACT}

This article is the result of a study conducted with a class of second module of the discipline of Physical Chemistry of a technical course in chemistry at a private school in the metropolitan area of Porto Alegre, RS. An experimental activity was carried out for the construction of concepts related to the quantities mass, volume and concentration. Tests were applied to assess knowledge before and after the development of this experimental activity. Past performance has been relatively poor, with a minimum grade 1.0, with a mean of 6.6. The results were statistically evaluated by applying analysis of variance, which revealed an improvement in the results of tests with $5 \%$ significance. The new

\footnotetext{
${ }^{1}$ Este artigo foi apresentado no IX Encontro Nacional de Pesquisa em Educação em Ciência, realizado em Águas de Lindoia, em 2013.
} 
results showed a minimum score equal to 4.0 and a mean of 8.9 .

Key words: mass, volume, concentration, quantities, difficulties, learning, experiences.

\section{INTRODUÇÃO}

A disciplina de Físico-Química é de suma importância para a formação do profissional Técnico em Química, pois os assuntos abordados funcionam como alicerce para que os mesmos possam, no decorrer da sua carreira, realizar as atividades pertinentes a sua habilitação, tais como as definidas na Resolução Normativa no 36 do Conselho Federal de Química no artigo 10: “Análise química e físicoquímica, químico-biológica, broma-tológica, toxicológica e legal, padronização e controle de qualidade (BRASIL, 1974).

Dentre um dos principais conteúdos desenvolvidos na disciplina de físico-química encontramos o preparo de soluções, nas formas de concentrações variadas, suas diluições e mistura. O conteúdo é desenvolvido em aulas expositivas com a realização de cálculos, utilizando diferentes fórmulas já definidas, para o entendimento e memorização dos mesmos. Souza e Santos (2008) definiram o ensino de físico-química como:

O ensino de Físico-Química praticado nas escolas de educação profissional e tecnológica do Brasil tem se caracterizado por uma prá-tica docente marcadamente tecnicista, cujas atividades se resumem à memorização de fórmulas, regras e/ou conceitos pré-estabelecidos ( SOUZA, SANTOS, 2008, p.1).

A memorização e o uso excessivo de fórmulas é uma das dificuldades observadas no ensino da disciplina (SILVA,2011), além de que, para o bom desenvolvimento da disciplina, o aluno necessita interdisciplinar coma matemática, outra disciplina das exatas que os alunos apresentam grande dificuldade de aprendizado.

Foi possível observar, no decorrer da abordagem do conteúdo "soluções" a dificuldade que os alunos apresentavam em diferenciar as grandezas massa, volume e concentração quando realizado as aulas expositivas.

Esses alunos, mesmo tendo a educação básica concluída, apresentaram dificuldades na interpretação de problemas, localização de dados e na diferenciação dos mesmos. Isto é, ao realizar leitura de exercícios propostos, os alunos não entendem o que foi solicitado e não conseguem identificar dados específico, como massa de soluto, volume de solução e concentração de solução.

Na formação de um técnico química, a elaboração de soluções, sua padronização e diluição são itens de extrema importância, uma vez que a realização de análises diversas faz parte da sua habilitação e, para que o mesmo possa realiza-la, ele precisa aprender a preparar soluções, ou pelo menos, diluir 
soluções já prontas. Desse modo, essas dificuldades representam um obstáculo importante a ser superado por parte dos professores, no processo de ensino.

$\mathrm{Na}$ educação matemática, tem sido estudado as metodologias para o ensino de grandezas, através da Teoria dos Campos Conceituais de Vergnand e das hipóteses didáticas que provêm das pesquisas desenvolvidas por Douady e Perrin-Glorian. (MORAES, FI-GUEIREDO e BELLEMAN, 2012).

Na educação em química, alguns autores (Giordam, Ferreira, Guimarães) tem estudado diversas metodologias relacionadas aos processos de aprendizagem dos conceitos científicos, uma vez que “aprender conceitos (...) é um processo lento, complexo e sempre inacabado, pois os conceitos vão sendo revistos e ampliados. (JUNIOR, LIMA, MAR-TINS, 2005, p.4 ). A construção de conceitos é um processo dialógico, onde o aluno necessita relacionar, confrontar novos conceitos com conceitos já aprendidos, apropri-ando-se então desse novo conceito.

Desse modo buscou-se utilizar a atividade experimental como ferramenta para a construção dos conceitos, uma vez que "a realização de atividades experimentais representam uma excelente ferramenta para que o aluno faça a experimentação do conteúdo e possa estabelecer a dinâmica e indissociável relação entre teoria e prática" (REGINAL-DO;SHEID, GÜLIICH, 2012,p.2)

Sendo assim, a experimentação tem papel importante na construção do conhecimento científico, não apenas pela atividade experimental, ou seja, pela observação dos fenômenos, mas devido as pesquisas e investigações que envolvem a atividade prática e que suscitam a construção de conceitos.

A experimentação proporciona discussões e problematizações de um tema ou experimento pelos alunos. A problematização pela equipe auxilia o aluno na construção do conhecimento, baseado nas observações e medições do experimento, acertos ou erros, e nas discussões em equipe acerca do mesmo. Desta forma deixamos de usar o experimento apenas como uma forma de reproduzir e comprovar o teórico.

Além disso, se tem defendido a importância da experimentação no ensino de Química por diversos autores, pois esse "constitui um recurso pedagógico importante que pode auxiliar na construção de conceitos” (FERREIRA; HARTWIG; OLIVEIRA, 2010, p. 101). A experimentação pode ser uma estratégia eficiente para a criação de problemas reais que permitam a contextualização e o estímulo de questionamentos para investigação (GUIMARÃES, 2009).

Com o objetivo de investigar como a experimentação em química impacta na compreensão das grandezas e na resolução de problemas, foi realizado um experimento com 12 estudantes do curso técnico em química de uma escola técnica privada da Grande Porto Alegre, RS, Brasil, para alunos com ensino médio já concluído.

Este artigo apresenta os resultados obtidos com testes aplicados antes e depois desse 
experimento, bem como a análise de variância dos resultados obtidos. A aplicação de análise de variância visa comprovar que o experimento apresentou resultados positivos.

Todos os doze alunos já haviam cursado o ensino médio regular e as disciplinas básicas do curso técnico em química, tais como: fundamentos de química, matemática, técnicas de laboratório. Sendo assim, os mesmos já possuíam conhecimentos prévios de química relativos ao curso técnico e as práticas de laboratório.

\section{METODOLOGIA}

O experimento compreendeu, além de pré e pós-testes, aulas expositivas dos conteúdos: tipos de soluções, grandezas e unidades de medidas, concentração de soluções que englobam: concentração comum (massa do soluto por volume de solução), Molaridade (quantidade de matéria do soluto por volume de solução), título (massa do soluto por massa da solução e volume de soluto por volume da solução), normalidade (equivalente grama do soluto por volume de solução) e densidade (massa do soluto por volume do soluto), atividades experimentais e questionário.

O trabalho foi realizado em cinco etapas:

Etapa 1: Iniciada com aulas expositivas, somando ao total três aulas, onde os alunos recebiam os conteúdos através de uma apostila e posteriormente o professor realizava as explicações referentes ao conteúdo, utilizando o quadro negro para a realização de exemplos e após foi solicitado aos alunos a realização de exercícios. Todos os exercícios foram realizados com conteúdos relativos aos conhecimentos prévios de química que os alunos possuíam e com o auxílio em tempo integral do professor.

Etapa 2: Realização de um teste com 10 questões de cálculos de concentração de soluções. Foram fornecidas as fórmulas, tabela periódica, calculadora e tabela de grandezas. As questões foram divididas da seguinte maneira: 3 questões para concentração massa de soluto por volume de solução (g/L),4 questões para concentração quantidade de matéria do soluto por volume de solução (mol/L),2 questões para concentração massa de soluto por massa de solução $(\mathrm{m} / \mathrm{m}), 1$ questões para concentração equivalente grama de soluto por volume de solução (eq.g/L). Em duas questões da concentração quantidade de matéria do soluto por volume de solução foi utilizado também a relação de densidade do soluto.

Etapa 3: A terceira etapa compreendeu a atividade experimental, na qual foram trabalhados os conceitos de propriedades físicas: massa, volume e densidade. Os alunos foram divididos em grupos de no máximo três componentes, receberam um cronograma com as etapas a serem desenvolvidas no laboratório, bem como uma revisão prévia dos conceitos de grandezas, unidades de medidas e concentração de soluções. 
Anteriormente à realização da atividade, o professor realizou um nova explanação dos conceitos presentes na atividade prática, buscando vincular os conhecimentos prévios dos alunos para que os mesmos pudessem desta forma realizar associações.

A atividade continha três experimentos distintos. Incialmente os alunos foram instruídos a realizar a pesagem de três volumes diferentes do reagente Etanol: 10ml, 30ml e 40ml, medidos previamente, pelo grupo, com o uso de uma proveta de 50mL.. Posteriormente, os mesmos deveriam realizar o cálculo da densidade do reagente, utilizando os dados obtidos, massa e volume. Foi realizado o mesmo experimento utilizando três pregos de tamanhos diferentes, onde para a medição de volume foi utilizado a técnica de deslocamento de um líquido. Sendo assim, após a pesagem dos pregos, os alunos deveriam colocar o mesmo em uma proveta que já continha um volume de água pré-definido, de forma que fosse possível observar o deslocamento do volume após a imersão do prego no líquido. Foi também realizado a densidade de quatro soluções de diferentes concentração com o soluto etanol, de forma que os mesmos pudessem observar a diferença entre a densidade do soluto e das soluções preparadas com o próprio soluto.

Posteriormente, os alunos foram questionados com relação aos conceitos de grandeza e de unidade de medida, densidade e relação da densidade do soluto e densidade da solução através das questões propostas no decorrer da atividade experimental: Quais as massas obtidas (utilizar o símbolo da grandeza e a unidade da mesma para responder a pergunta)? Quais os volumes medidos (utilizar o símbolo da grandeza e a unidade da mesma para responder a pergunta)?Para todas as três medidas foi encontrado o mesmo valor de densidade? Justifique.

O objetivo da atividade experimental foi o de tirar o aluno da condição passiva de mero ouvinte ou o de realizador de atividades motoras e colocá-lo frente situações problema, para que o mesmo pudesse construir os conceitos através dos questionamentos e a inter-relação do conteúdo prático e os conteúdos teóricos trabalhados anteriormente.

Etapa 4: Foram aplicados novamente as mesmas questões aplicadas no pré-teste, apenas modificados os valores fornecidos. Foram fornecidas novamente as fórmulas, tabela periódica, calculadora e tabela de grandezas.

Etapa 5: A quinta etapa do trabalho compreendeu a análise dos resultados obtidos com os testes aplicados na segunda e na quarta etapas. Os resultados foram analisados com a aplicação de análise de variância, considerando os dois testes como "tratamentos" e as notas de cada aluno como "repetições".

A hipótese nula consistiu na suposição de que os resultados obtidos com as duas provas não apresentariam diferenças significativas e, portanto, o trabalho de reforço não teria surtido efeito. A hipótese alternativa consistiu, portanto, na suposição de que o trabalho de complementação surtiu efeito e que a média dos resultados obtidos na segunda prova se apresentaria significativamente diferente da 
média dos resultados da primeira prova.

\section{ANÁLISE E INTERPRETAÇÃO DOS RESULTADOS}

No presente trabalho foram avaliados os dados quantitativos referentes as notas apresentados pelos alunos no pré e no pós-teste, conforme descrito anteriormente, utilizando a análise de variância como método estatístico para a avaliação do efeito da atividade prática executada entre os testes. Os dados qualitativos referentes aos questionamentos das atividades experimentais serão avaliados em outro artigo.

A Tabela 1 mostra em suas duas primeiras colunas as notas dos alunos nos dois testes aplicados. A coluna N1 mostra os resultados do primeiro teste, realizado antes da atividade prática. A coluna N2 mostra os resultados do segundo teste, aplicado após o trabalho de complementação.

Observa-se o evidente resultado positivo sobre as notas, onde nenhum aluno obteve na segunda avaliação nota inferior à primeira. As notas do primeiro teste tiveram nota mínima igual a 1,0 e houve uma nota 10,0, com média igual a 6,6 . No segundo teste, a nota mínima foi 4,0 e houve cinco notas 10,0 , com média igual a 8,9 .

As colunas seguintes fazem parte da análise de variância. Cada nota é decomposta como uma soma da média geral de todas as notas, do efeito dos tratamentos e do termo aleatório. As duas colunas seguintes mostram justamente a média geral, depois aparecem (nas duas colunas subsequentes) os efeitos dos tratamentos e, por fim, a matriz (nas duas últimas colunas) com os termos aleatórios.

Tabela 1: Resultados obtidos e decomposição dos dados para análise de variância.

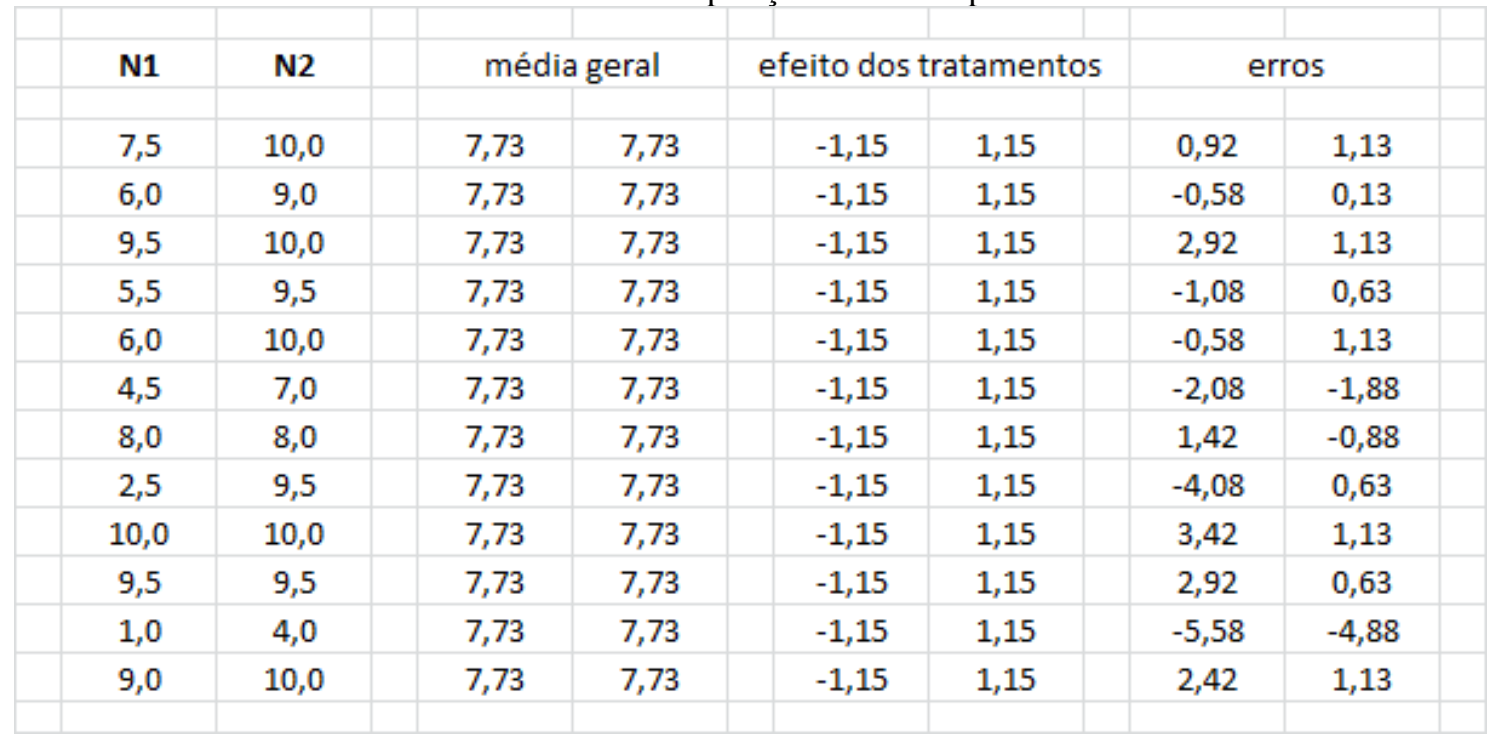

Cada um dos componentes das duas primeiras colunas pode ser escrito como a soma dos componentes correspondentes nas colunas seguintes. Por exemplo, a primeira nota do teste N1, 7,5, é 
escrita como a soma da média geral, 7,73, do efeito dos tratamentos, -1,15, nesse caso, e do termo aleatório, 0,92. Cada uma das outras notas pode ser escrita com a mesma decomposição.

A Tabela 2 apresenta o resultado da análise de variância. As fontes de variação são os tratamentos, ou seja, os dois testes, e os erros. Os tratamentos têm 1 grau de liberdade, enquanto os erros apresentam 22 graus de liberdade.

Tabela 2: Resultado obtido com a análise de variância.

\begin{tabular}{|c|c|c|c|c|}
\hline fonte & g.l. & sq & qm & F \\
\hline trat & 1 & 31,510 & 31,510 & 5,459 \\
\hline erros & 22 & 126,979 & 5,772 & \\
\hline & & & & \\
\hline & & & F tab & 4,3009 \\
\hline & & & & \\
\hline & & & & rejeita \\
\hline
\end{tabular}

As colunas seguintes apresentam as somas dos quadrados e os quadrados médios. A soma dos quadrados dos tratamentos é obtida pela soma dos quadrados das colunas, na Tabela 1, correspondentes aos efeitos dos tratamentos. A soma dos quadrados dos erros é obtida pela soma dos quadrados das colunas, na Tabela 1, correspondentes aos erros.

Os quadrados médios são obtidos da razão das somas dos quadrados para os graus de liberdade. O quadrado médio dos tratamentos é obtido pela divisão da soma dos quadrados dos tratamentos pelo respectivo número de graus de liberdade. O quadrado médio dos erros é obtido pela divisão da soma dos quadrados dos erros pelo respectivo número de graus de liberdade.

O valor calculado de F é obtido pela divisão do quadrado médio dos tratamentos pelo quadrado médio dos erros. O valor calculado de $\mathrm{F}$ deve ser comparado com o valor tabelado, obtido da distribuição para F correspondente ao valor de significância adotado.

Para uma significância de 5\%, observa-se que o $\mathrm{F}$ tabelado é inferior ao $\mathrm{F}$ calculado. Esse resultado sugere que a hipótese nula seja rejeitada. Em outras palavras, as médias das duas provas se mostram diferentes, com significância de 5\%.

Em outras palavras, ainda, é possível afirmar que existe diferença significativa entre as médias obtidas nas duas provas, com probabilidade de erro (nessa afirmação) de até $5 \%$.

Seria possível aplicar testes específicos para avaliar qual das médias é superior. Quer dizer, seria possível aplicar testes, como o teste de Duncan, que mostrassem que a média do conjunto de notas N2 é superior à média do conjunto de notas N1.

Entretanto, basta uma inspeção visual nos resultados para perceber que as notas N2 são 
superiores às notas N1 e que existe uma diferença razoável entre os valores obtidos pelos alunos nos dois testes. O resultado da análise de variância confirma essa diferença e mostra com que significância ocorre essa diferença.

\section{CONSIDERAÇÕES FINAIS}

O presente trabalho teve como objetivo mostrar as dificuldades encontradas pelo professor para o desenvolvimento de um conteúdo relativo a disciplina de físico-química e também o de comprovar que as atividades experimentais auxiliam na construção de conceitos.

Desta forma deixamos de usar o experimento apenas como uma forma de reproduzir e comprovar o teórico. O aluno é posto frente a questionamentos e utiliza do experimento para resolvêlos, investigando, envolvendo-se, questionando, desenvolvendo as habilidades de comunicação, desta forma o aluno passa a ter o papel principal na construção do seu próprio conhecimento, deixando de ser apenas um mero ouvinte e coadjuvante.

A partir de uma análise dos resultados de pré e pós teste foi possível observar um crescimento quantitativo nas notas dos alunos. A atividade experimental, embora muito simples, trabalhou conceitos importantes para o entendimento dos conteúdos da disciplina. Na atividade os alunos foram conduzidos a problematizar os conceitos com os colegas do grupo, formular ideias e testar hipóteses. Desta forma, puderam construir o conhecimento baseado nas suas próprias observações e nos conhecimentos prévios.

A realização de uma aula teórica anteriormente a aula prática foi de suma importância, pois foi possível apresentar a teoria anteriormente para que os alunos tivessem um embasamento teórico prévio e como auxiliar nas seus questionamentos e hipóteses. Desta forma "a relação entre a teoria e a prática é uma via de mão-dupla, na qual se vai dos experimentos à teoria e das teorias aos experimentos, para contextualizar, investigando, questionando, retomando" (SILVA e ZANON apud REGINALDO,SHEID,GÜLIICH, 2012, p.11)

Através da leitura propostas nas aulas teóricas os alunos enriquecem seus argumentos quando são colocados frente a estes questionamentos e quando estão discutindo em um grupo os dados obtidos através da atividade experimental, favorecendo o entendimento do caráter social da ciência e também como forma de socializar os alunos (REIGOZA e JIMENEZ apud GONÇALVES, 2006, p.228)

Também como para Ausubel, que na sua teoria defende "a transmissão de conhecimentos por parte do professor pode ser um método adequado e eficaz, sempre e quando leva em conta os conhecimentos prévios dos alunos e sua capacidade de compreensão" (A-SUBEL apud CARRETO, 1997,p.17). Desta forma buscou-se, na etapa 1, apresentar o conteúdo de forma organizada proporcionando a contextualização e problematização com os alunos. 


\section{REFERÊECIAS}

JUNIOR, O. A.; LIMA,M. E. C. C. ; MARTINS, C. C. A Formação de Conceitos Científicos: Reflexões a Partir da Produção de uma Coleção de Livros Didáticos. V Encontro Nacional de Pesquisa em Educação em Ciências. 2005. Bauru, Atas do V ENPEC.

AUSUBEL,D.P.; NOVACK,J.D, HANESIAN, H. Educational psychology. N. York: Holt, Rinehart e Winston, 1978. Apud: CARRETO, M. C . Construtivismo e Educação. Porto Alegre. Artes Médicas, 1977. 96p.

BRASIL. Conselho Federal de Química. Resolução Normativa N 36, Brasília,1974.

FERREIRA, L.H.; HARTWIG, D. R.; OLIVEIRA, R. C. Ensino Experimental de Química: Uma Abordagem Investigativa Contextualizada. Química Nova na Escola, São Paulo, v. 32, n. 2, p. 101 a $106,2010$.

GIORDAN, M., O Papel da experimentação no ensino de ciências. Química Nova na Escola, n 10, p43-49, 1999.

GUIMARÃES, C.C.. Experimentação no Ensino de Química: Caminhos e Descaminhos Rumo à Aprendizagem Significativa. Química Nova na Escola, São Paulo, v. 31, n. 3, p. 198 - 202. agosto 2009.

GONÇALVES, F.P.;MARQUES,C.A. Contribuição Pedagógica e epistemológica em textos de experimentação no ensino de química. Investigação em Ciência, V11(2), pp. 219-238, 2006.

MORAES, L. B.; FIGUEIREDO, A. P. N. B. ; BELLEMAIN, P. M. B. Estudo das Situações de volume como grandeza no ensino médio sob a ótica da teoria dos campo conceituais. VII Encontro Paraibano de Educação Matemática - EPBEM, 2012. João Pessoa. Anais do Encontro Paraibano de Educação Matemática(2012) - Volume 1, Número 1.

REGINALDO, C. C. ;SHEID, N. J.; GÜLIICH, R.I.C. O Ensino de Ciências e a Experimentação. In Seminário de Pesquisa em Educação da Região Sul. 9. 2012. Caxias do Sul. Anais IX ANPED SUL.

SILVA J. F S.S. José Fabiano Serafim da Silva. Concentração de Soluções: A dificuldade de interpretação das grandezas massa e volume. In Conferência Interamericana de Educação Matemática. 13. 2011. CIAEM. 2011.

SOUZA, F. L.; SANTOS, A.C.S. Interdisciplinaridade e Ensino de Físico-Química para os Cursos de Agropecuária e Agroindústria da EAF-Codó/MA. In. Encontro Nacional de Ensino de Química. 14. 2008. Curitiba. Anais XIV ENEQ. 